\title{
Role of distinct surfaces of the G9a ankyrin repeat domain in histone and DNA methylation during embryonic stem cell self-renewal and differentiation
}

\author{
Danielle Bittencourt ${ }^{*}$ Brian H Lee, Lu Gao, Daniel S Gerke and Michael R Stallcup*
}

\begin{abstract}
Background: Epigenetic modifications such as histone and DNA methylation are essential for silencing pluripotency genes during embryonic stem cell (ESC) differentiation. G9a is the major histone H3 Lys9 (H3K9) methyltransferase in euchromatin and is required for the de novo DNA methylation of the key regulator of pluripotency Oct3/4 during ESC differentiation. Surprisingly, the catalytic activity of G9a is not required for its role in de novo DNA methylation and the precise molecular mechanisms of G9a in this process are poorly understood. It has been suggested that the G9a ankyrin repeat domain, which can interact with both H3K9me2 and the DNA methyltransferase DNMT3A, could facilitate de novo DNA methylation by bridging the interaction between DNMT3A and H3K9me2-marked chromatin.
\end{abstract}

Results: Here, we demonstrate that the G9a ankyrin domain H3K9me2-binding function is not required for the de novo DNA methylation of Oct3/4 during ESC differentiation. Moreover, we show that the interaction between the G9a ankyrin domain and DNMT3A is not sufficient to ensure efficient de novo DNA methylation. More importantly, we characterize a specific residue of the G9a ankyrin domain (Asp905) that is critical for both maintaining cellular H3K9me2 levels in undifferentiated ESCs and for the establishment of de novo DNA methylation during differentiation.

Conclusions: These results represent an exciting breakthrough, which reveals 1) an unexpected critical biological function of the G9a ankyrin domain in global histone H3K9 methylation and 2) valuable insights into the molecular mechanisms and interaction surfaces through which G9a regulates de novo DNA methylation of Oct3/4 during ESC differentiation.

Keywords: Histone methylation, DNA methylation, Embryonic stem cell, G9a, Ankyrin, H3K9, DNMT3A, Oct3/4

\section{Background}

The remarkable property of embryonic stem cells (ESC) to remain in a pluripotent state, poised to execute a broad range of developmental programs, is due to fine control of proper gene expression patterns by key regulators of pluripotency such as the Oct $3 / 4$ and Nanog transcription factors. This pluripotent state is characterized by maintenance of transcriptionally permissive chromatin, which underlies high genome plasticity from

\footnotetext{
*Correspondence: daniellebittencourt@hotmail.com; stallcup@usc.edu From the Department of Biochemistry and Molecular Biology, Norris Comprehensive Cancer Center, University of Southern California, NOR 6314 1441 Eastlake Avenue, Los Angeles 90089-9176, CA, USA
}

which other gene expression patterns are derived during development [1-4]. Epigenetic modifications such as histone and DNA methylation are essential for chromatin reorganization during development, resulting in the silencing of early embryonic genes and activation of lineagespecific genes that drive differentiation in cell fates [5].

Underlining the importance of histone and DNA methylation in the control of proper gene expression patterns in ESCs, disruption of the G9a histone methyltransferase gene in mice results in growth retardation and early embryonic lethality due to deregulation of developmental genes [6]. G9a-null ESCs display a dramatic reduction in cellular mono- and dimethylated histone H3 lysine K9 
(H3K9) levels, indicating its role as the major histone H3K9 methyltransferase. Furthermore, G9a-null ESCs show deficient de novo DNA methylation of several early embryonic genes, including Oct3/4 and Nanog during differentiation [7-9], suggesting a key role of G9a in establishing de novo DNA methylation. However, in contrast to histone methylation, the catalytic function of the G9a C-terminal SET domain (Figure 1A, top panel) is not required for its role in de novo DNA methylation $[7,10]$.

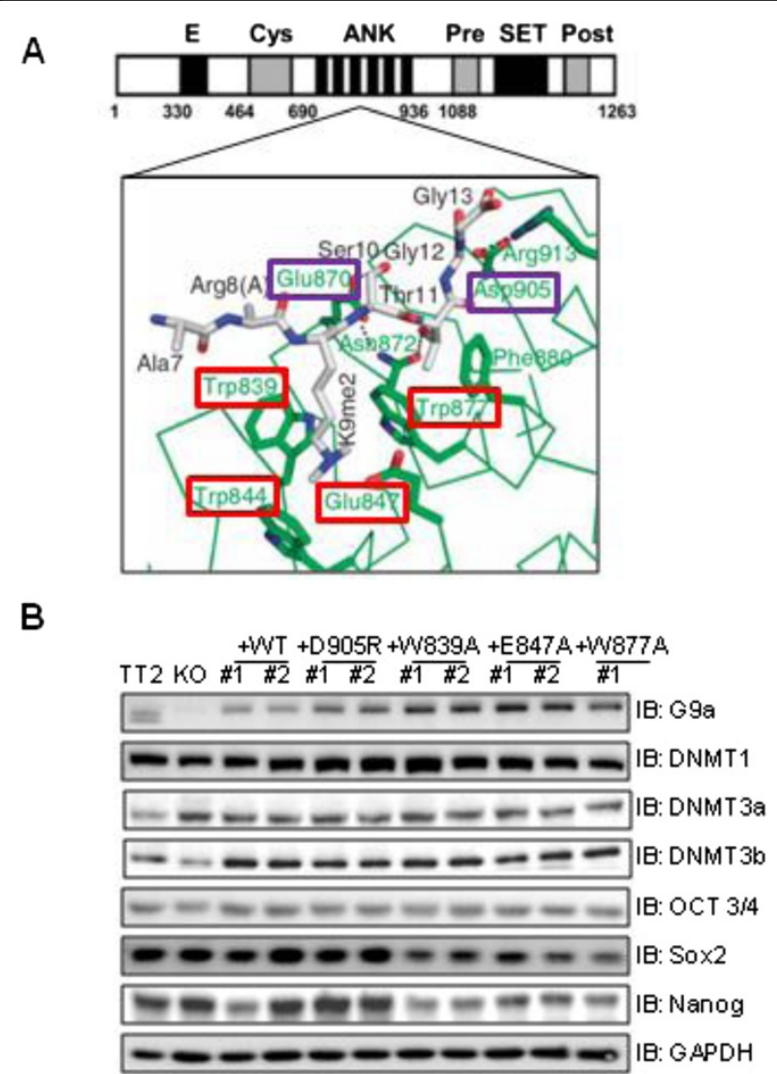

Figure 1 Characterization of mouse embryonic stem (ES) clonal cell lines expressing G9a bearing point mutations in the cage and non-cage residues of the ankyrin repeat (ANK) domain. (A) Top panel: Diagram of full-length mG9a showing amino acid sequence numbers and specific domains: $E$, Glu-rich; Cys, Cys-rich ring finger-like; ANK, ankyrin repeat; SET, methyltransferase; and Pre and Post, Cys-rich Pre-SET and post-SET. Bottom panel: Schematic representation of the structural basis for ankyrin repeat (green) recognition of dimethylated histone H3 Lysine 9 (gray). G9a residue numbers are shown. H3K9me2 binds in a partial hydrophobic cage composed of residues highlighted in red. Peptide binding is further specified by the interaction of non-cage G9a residues (highlighted in purple) with H3 Ser10, Thr11, Gly12 and Gly13. (B) Immunoblots (IB) were performed on the indicated proteins (with GAPDH as loading control) using whole cell extracts derived from wild-type (TT2) mouse embryonic stem cells (mESCs), G9a-null mESCs (knock-out; $\mathrm{KO}$ ) or distinct clonal ESC lines (indicated by \#1 and \#2) stably expressing wild-type G9a (+WT) or G9a harboring the indicated point mutations in the ANK domain.
A recent report has shown that the deletion of the G9a central ankyrin repeat domain (ANK, Figure 1A, top panel) results in impaired de novo DNA methylation of the Oct3/4 gene promoter during ESC differentiation [7]. Interestingly, the G9a ANK domain also binds to the de novo DNA methyltransferase DNMT3A in vitro [7], although it has not yet been determined whether this interaction is required and/or sufficient for G9a-mediated de novo DNA methylation. Furthermore, the G9a ANK domain specifically binds to the product of G9a catalytic activity: histone H3 mono- or dimethylated at K9 [11]. Indeed, G9a binding to K9-methylated histone $\mathrm{H} 3$ involves recognition of two different features of the methylated histone by a discreet region of the ANK domain, as previously demonstrated by X-ray crystallography: 1) a partial hydrophobic cage of ANK recognizes the dimethylamino moiety of $\mathrm{H} 3 \mathrm{~K} 9 \mathrm{me} 2$, and 2) a nearby non-cage functional surface of ANK interacts with residues 10 to 13 of histone H3 [11] (Figure 1A, bottom panel). However, the biological function of this binding is not fully understood, and to date, the precise molecular mechanisms by which G9a and, in particular its ANK domain, contribute to de novo DNA methylation remain unclear.

To address these questions, we established clonal ESC lines expressing G9a with point mutations in the ANK domain that prevent K9-dimethylated histone H3 binding. With these cell lines, we investigated the role of this histone $\mathrm{H} 3$ binding surface in regulating markers of ESC differentiation, global cellular H3K9 methylation, and de novo DNA methylation of the Oct3/4 gene promoter both in undifferentiated ESCs and during retinoic acid (RA)-induced differentiation. Our results reveal that these three parameters of ESC biology have distinct requirements for different surfaces of G9a. Surprisingly, we found that different point mutations that disrupt ANK domain binding to K9-dimethylated histone H3 had different phenotypes, suggesting that the histone H3 binding surface of the ANK domain may have multiple functions involving multiple interacting protein partners. We also define a specific residue in the G9a ANK domain that is critical for its role in de novo DNA methylation. Altogether, our findings represent an exciting breakthrough, which can reveal 1) novel biological functions of the G9a ANK domain, and 2) valuable insights into the mechanisms of the distinct functional surfaces of the G9a ANK in supporting de novo DNA methylation, H3K9 methylation and ESC differentiation.

\section{Results}

Derivation of clonal embryonic stem cell lines expressing G9a with point mutations in the ankyrin repeat domain Our previous X-ray crystallography studies characterized six residues of the G9a ankyrin repeat (ANK) domain that are involved in G9a binding to the N-terminal tail 
of histone H3 that is mono- or dimethylated at K9 [11]. While four of these residues (Trp839, Trp844, Glu847 and Trp877) form a partial hydrophobic cage that interacts with the dimethylamino moiety of H3K9me2 (Figure 1A, bottom panel, highlighted in red), residues Glu870 and D905 of the G9a ANK non-cage functional surface interact with amino acids 10 to 13 of histone H3 [11] (Figure 1A, bottom panel, highlighted in purple). A single point mutation in any of these six G9a residues is sufficient to disrupt the interaction in vitro between G9a and a histone $\mathrm{H} 3 \mathrm{~N}$-terminal peptide dimethylated at $\mathrm{K} 9$. Importantly, none of these mutations abolish G9a catalytic function as determined by in vitro assays or its structural integrity, as demonstrated by the ability of the G9a ANK domain containing any of these mutations to interact with protein partners GRIP1 and Gfi1 [11]. Thus, to date the biological function of the histone H3 interacting surface of the G9a ANK domain is unknown.

In order to investigate whether the methylhistone $\mathrm{H} 3$ binding function of the G9a ANK domain is required for mESC differentiation and the accompanying de novo DNA methylation of key pluripotency genes, G9a-null
mESCs (knock-out; KO) were used to generate stable clonal cell lines expressing either wild-type (+WT) G9a or G9a containing various ANK domain point mutations mentioned above. Importantly, two independent clones expressing each G9a mutant were established in order to control for potential clonal artifacts. Immunoblots were performed to verify that the G9a expression level in these cell lines is comparable to the endogenous G9a expression in the wild-type parental mESC line TT2 (Figure 1B, top panel). Importantly, the protein expression levels of master regulators of pluripotency such as Oct3/4, Sox2 and Nanog (Figure 1B), as well as of key players in DNA methylation (DNA methyltransferases: DNMT3A, DNMT3B and DNMT1), were also comparable in all undifferentiated ESC lines (Figure 1B). Immunoblots were repeated several times using different protein extracts and slight differences observed in protein expression levels between cell lines seen here (Figure 1B) were not found consistently. Notably, messenger RNA (mRNA) expression levels of early embryonic genes (Oct3/4, Nanog, Klf4, Esrrb and Dppa3) determined by quantitative RT-qPCR were similar in all cell lines (Figure 2B).
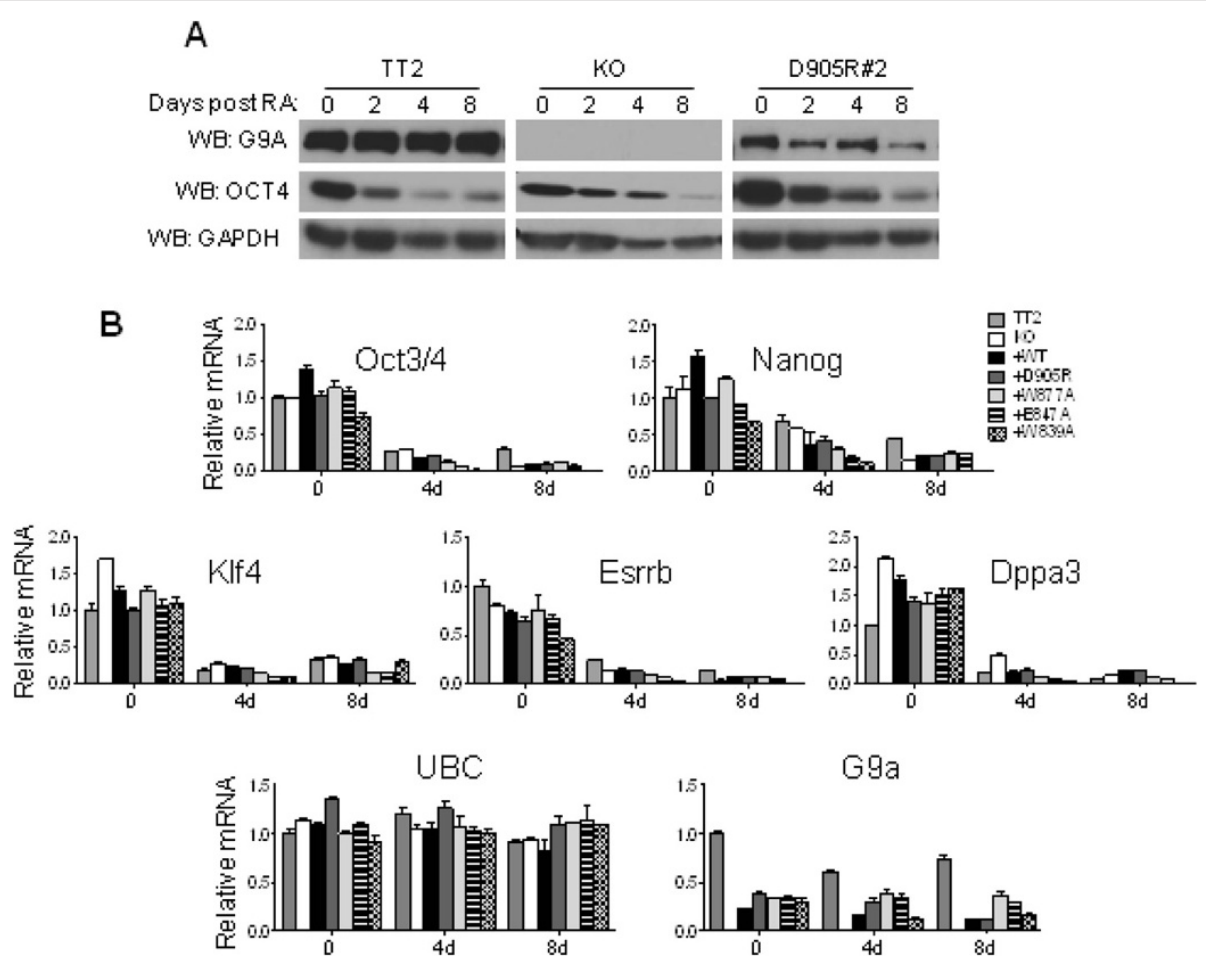

Figure $2 \mathrm{G9a}$ is not required for the transcriptional repression of selected early embryonic genes during retinoic acid (RA)-induced differentiation. The indicated mESC lines were untreated (0) or treated with $1 \mu \mathrm{M}$ RA for 2, 4 or 8 days. (A) Immunoblots were used to determine G9a, Oct3/4 and GAPDH (loading control) protein levels in whole cell extracts. (B) Graph shows the messenger (mRNA) levels for the indicated genes using RT-qPCR analysis with Ubiquitin $C(U B C)$ as the normalization control. All mRNA levels are normalized to the TT2 0 RA sample. Results shown are mean \pm SD for three PCR reactions performed on the same CDNA sample and are representative of three independent experiments. 
A specific histone $\mathrm{H} 3$ binding surface of the $\mathrm{G} 9 \mathrm{a}$ ankyrin repeat domain is required for establishing de novo DNA methylation during mouse embryonic stem cell differentiation

Several reports demonstrate the requirement of G9a and its ANK domain for proper de novo DNA methylation of numerous early embryonic genes [6-8,12]. However, the molecular mechanisms of G9a ANK domain function in facilitating DNA methylation remain unknown. Using the ESC clones expressing the G9a point mutants described above, we investigated the role of the methylhistone binding surface of the G9a ANK domain in the de novo DNA methylation of the Oct3/4 gene promoter during ESC differentiation. Differentiation of stable ESC lines expressing wild-type or mutant G9a was induced by the addition of retinoic acid (RA) to the growth medium. The DNA methylation status of the Oct3/4 proximal promoter region prior to and after 8 days of RA treatment (Figure 3) was assessed by sequencing PCR products generated from bisulfite-treated genomic DNA. As expected, in undifferentiated mESCs, the Oct3/4 gene promoter is not methylated (Figures $3 \mathrm{~B}$ and $3 \mathrm{C}$ ). In agreement with previous findings, mESCs expressing either endogenous wild-type G9a (TT2) or exogenously introduced wild-type G9a (+WT) underwent de novo DNA methylation of the Oct3/4 promoter, with CpG methylation levels of 46 to $58 \%$ in the region examined after 8 days of RA treatment (Figures 3B and 3D, lower

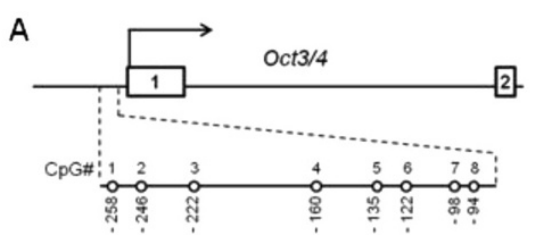

B

\begin{tabular}{|c|c|c|c|c|}
\hline \multicolumn{5}{|c|}{ DNA Methlation (\%) } \\
\hline \multirow{2}{*}{$\begin{array}{c}\text { Clonal } \\
\text { Cell line }\end{array}$} & \multicolumn{2}{|c|}{ Prior to RA } & \multicolumn{2}{|c|}{8 days RA } \\
\cline { 2 - 5 } & $\# 1$ & $\# 2$ & $\# 1$ & $\# 2$ \\
\hline$\pi 2$ & 0 & & 53 & \\
\hline KO & 1 & & 26 & \\
\hline+ WT & & 3 & 58 & 46 \\
\hline +D905R & 1 & 5 & 21 & 23 \\
\hline +E847A & 0 & 0 & 80 & 80 \\
\hline +W839A & 0 & 0 & 79 & 47 \\
\hline +W877A & 0 & & 62 & \\
\hline
\end{tabular}

c

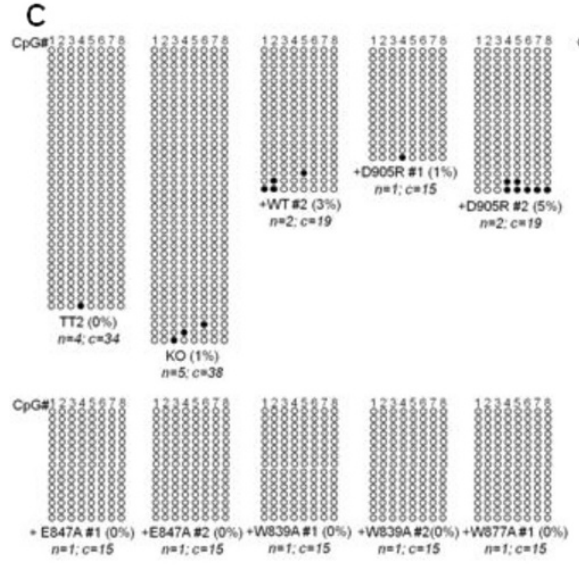

D

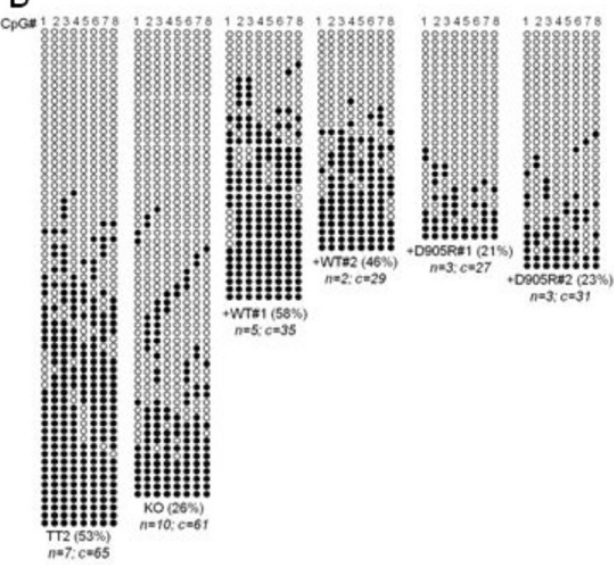

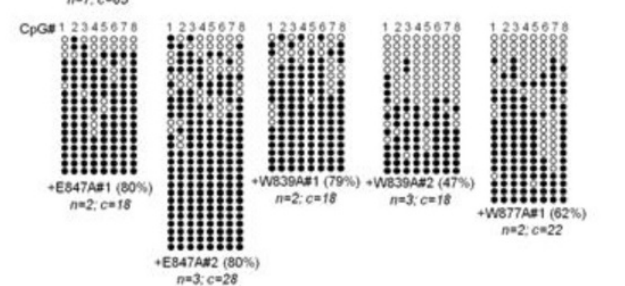

Figure 3 The G9a non-cage histone $\mathrm{H} 3$ binding surface of the ankyrin repeat (ANK) domain is required for de novo DNA methylation of the Oct3/4 gene promoter during retinoic acid (RA)-induced embryonic stem cell (ESC) differentiation. DNA extracted from the indicated mouse embryonic stem cell (mESC) lines, grown under self-renewal conditions or treated with RA for 8 days to induce differentiation, was treated with sodium bisulfite, amplified using specific Oct3/4 promoter primers, cloned and subjected to sequence analysis. (A) Schematic diagram of CpG positions within the Oct3/4 gene promoter is shown. The methylation status of eight CpG sites (no. 1 to 8 ) within the promoter (-90 to -258 relative to the transcription start site) was examined. (B) Percentage of DNA methylation for the Oct3/4 promoter region encompassing eight CpG sites of each clonal cell line is indicated in the table, which summarizes data shown in (C) and (D). DNA extracted from the indicated mESC lines that were untreated (C) or treated with RA for 8 days (D) was treated with sodium bisulfite, amplified using specific Oct3/4 promoter primers, cloned and subjected to sequence analysis. Each horizontal row represents results from sequencing a separate DNA clone. The overall percentage of methylated CpG sites is indicated in parentheses beside the clone designation. The number of independent biological replicate experiments ( $n$ ) from which the total number of DNA clones (c) were derived is also indicated. 
panel). Point mutations in key hydrophobic cage residues of the G9a ankyrin domain that specifically recognize the dimethylamino moiety of H3K9me2 (+W839A, +W877A and + E847A) [11] did not compromise de novo DNA methylation of Oct3/4 (Figure $3 \mathrm{~B}$ and $3 \mathrm{D}$ ). These results suggest that G9a binding to histone H3 containing mono- and dimethyl $\mathrm{K} 9$ is not required for de novo DNA methylation. In contrast, mESCs expressing G9a harboring a single point mutation of the Asp905 residue located in a distinct non-cage surface of the ANK domain that interacts with histone $\mathrm{H} 3$ residues 10 to 13 [11] display more than a twofold reduction in de novo DNA methylation levels of Oct3/4, similar to the levels observed in G9a-null mESCs (knock-out; KO) (Figure 3B and $3 \mathrm{D})$. Although some degree of clonal variability was observed between the two W839A mutant clones, both clones have higher DNA methylation levels after 8 days of RA treatment compared to the KO and D905R. Therefore, we can conclude that the W839A mutation does not impair de novo DNA methylation of Oct4. In summary, these results indicate that the cage and non-cage portions of the dimethyl-histone $\mathrm{H} 3$ binding surface have common and distinct functions, that is, binding of histone $\mathrm{H} 3$ containing dimethyl $\mathrm{K} 9$ and regulation of de novo DNA methylation, respectively.

\section{The G9a ankyrin repeat domain, but not its histone- binding surfaces, is required for the interaction between G9a and DNMT3A}

Previous studies have shown that the G9a ANK domain is required in vitro for binding between G9a and DNMT3A [7]. We confirmed these findings via coimmunoprecipitation of transiently transfected human influenza hemagglutinin (HA) tagged full-length WT or $\triangle \mathrm{ANK}$ mutant mouse G9a and full-length mouse DNMT3A in a different cell line (COS-7 cell extracts) (Figure 4B). Although the interaction with DNMT3a is observed with WT G9a, it is abrogated in the G9a $\triangle \mathrm{ANK}$ mutant (Figure $4 \mathrm{~B}$, left panel, lane 3 ). However, the specific functional surface of the G9a ANK domain responsible for this binding, as well as the physiological relevance of this interaction for establishing de novo DNA methylation, has not been defined. In order to determine whether the impaired de novo DNA methylation observed in the D905R non-cage G9a ANK mutant ESC lines is due to compromised interaction between DNMT3A and the mutant G9a, co-immunoprecipitation of transiently transfected FLAG-tagged full-length (WT or mutant) mouse G9a and full-length mouse DNMT3A was performed in COS-7 cell extracts. As expected, coimmunoprecipitated DNMT3A was detected when antibodies against FLAG were used for immunoprecipitation of wild-type G9a (Figure 4A, left panel, lane 3). Interestingly, none of the mutations in the G9a ANK domain eliminated binding between G9a and DNMT3A (Figure 4A, left panel lane 3). Anti-FLAG and Anti-G9a immunoblots on the same samples (Figure 4A, right and middle panels, respectively) are shown as a control for G9a immunoprecipitation. Although the efficiency of DNMT3A co-precipitation varied among the clones, the pattern of variation did not correlate with the observed DNA methylation of the Oct3/4 promoter; that is, DNMT3A co-precipitation with the D905R mutant (which has reduced DNA methylation of Oct3/4) is equal to or better than that for several of the other mutants (which support wild-type DNA methylation of Oct3/4). These results suggest that G9a binding to DNMT3A via the G9a ANK domain does not involve the ANK histone $\mathrm{H} 3$ binding surface but rather a different part of the ANK domain. More importantly, these data show that, although G9a and DNMT3A interaction is maintained in the D905R non-cage ANK mutant, this interaction is not sufficient to ensure proper de novo DNA methylation and a non-redundant function of the noncage surface of the ANK H3 binding domain is critical to this process.

\section{The G9a non-cage ankyrin repeat domain histone-binding surface is required to maintain global cellular $\mathrm{H} 3 \mathrm{~K} 9 \mathrm{me} 2$ levels}

The G9a C-terminal SET catalytic domain is responsible for its H3K9 methyltransferase activity [13]. As shown previously [6], we found that global H3K9me2 levels were dramatically decreased upon G9a depletion (Figure 4C, lane 2). However, these levels in mESCs were not affected by mutations in the G9a ankyrin cage domain (Figure $4 \mathrm{C}$, lanes 7 to 11 ). Surprisingly, a dramatic decrease in $\mathrm{H} 3 \mathrm{~K} 9 \mathrm{me} 2$ levels was observed in mESCs harboring the G9aD905R mutation in the noncage histone $\mathrm{H} 3$ binding surface of the ANK domain (Figure 4C, lanes 5 and 6). The H3K9me2 level was similar in cells expressing G9aD905R or lacking G9a altogether, despite the fact that the G9a D905R mutant is not catalytically dead when assayed in vitro [11]. It is well known that G9a exerts its histone methyltransferase activity in a heterodimeric complex with the G9a-like protein GLP [14]. In order to rule out the possibility that the impaired histone methylation observed in mESCs expressing G9aD905R (Figure 4C, lanes 5 and 6) was due to the fact that the D905R mutation in the G9a ANK domain abrogates the interaction between G9a and GLP, co-immunoprecipitation of transiently transfected FLAG-tagged full-length (WT or D905R mutant) mouse G9a and HA-tagged full-length mouse GLP was performed in COS-7 cell extracts. Co-immunoprecipitated GLP was detected when antibodies against FLAG were used for immunoprecipitation of wild-type G9a (Figure 4D, left panel, 

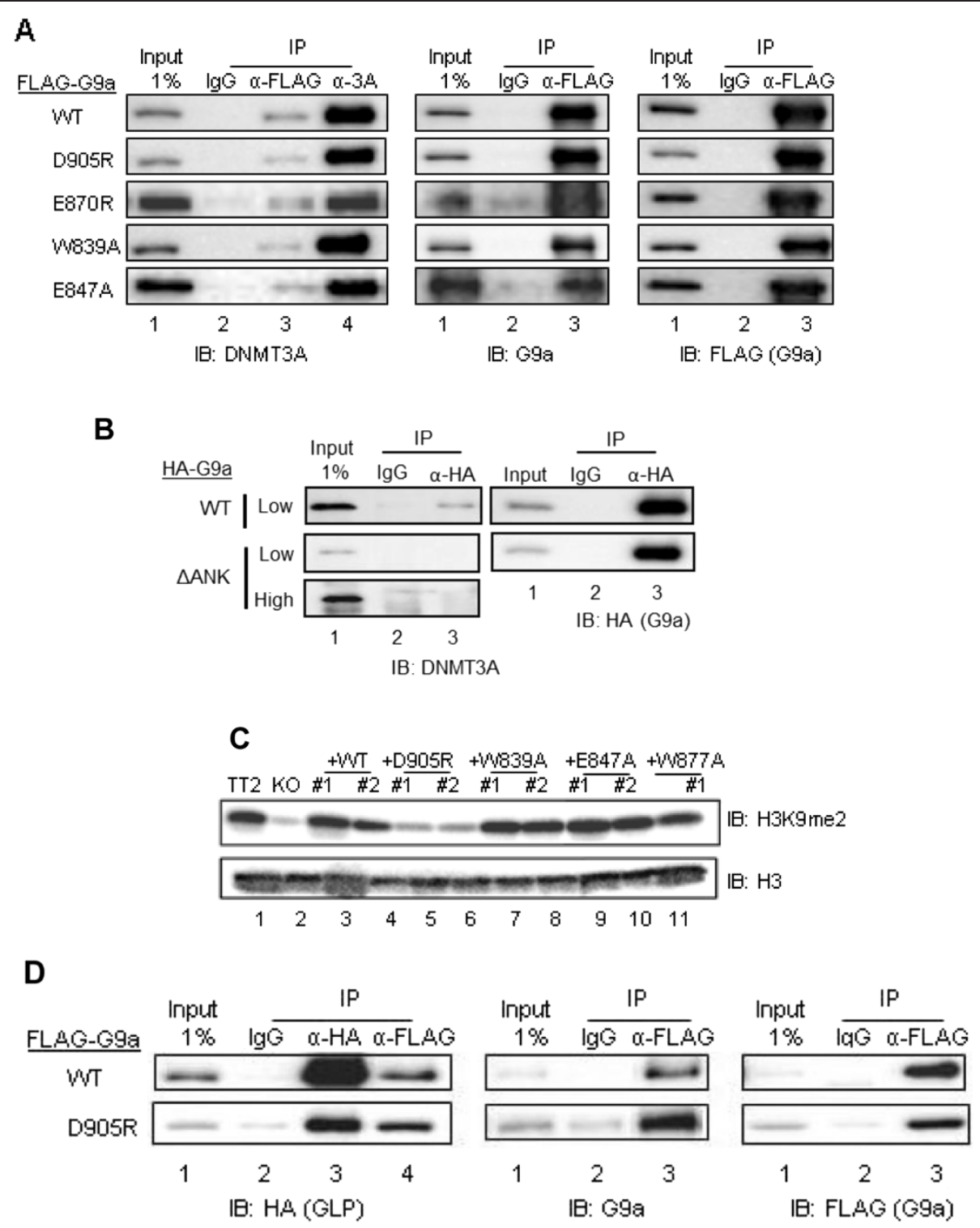

Figure 4 Mutations in the histone $\mathrm{H} 3$ binding surface of the G9a ankyrin repeat (ANK) domain do not abrogate binding to DNMT3A. COS- 7 cells were transiently transfected with pcDNA3.1-mDNMT3A (A and B) or pSG5-HA-mGLP full-length (D) and pSG5-FLAG-mG9a full-length (A, B and D) or pSG5-HA-mG9a. $\triangle A N K$ (B). G9a was either wild-type (WT) or contained the indicated point mutations in the ANK domain. G9a, GLP and DNMT3A were immunoprecipitated from cell extracts with an anti-FLAG/anti-HA, anti-HA or anti-DNMT3A antibody, respectively. Non-immune IgG antibody was used for immunoprecipitation background estimation. Bound proteins were analyzed by immunoblot (IB) with the indicated antibodies. A 1\% input sample was loaded for comparison. High (3 minutes) and low (1 minute) exposure times are shown (B). (C) Immunoblots were used to determine H3K9me2 and H3 protein levels in acid histone extracts from the indicated undifferentiated mESC lines.

lane 4). These results indicate that G9a binding to GLP is not abrogated by the D905R mutation in the G9a ANK domain. Altogether, these findings reveal a novel role of the G9a ANK domain in global histone H3K9 methylation and support the hypothesis where the Asp905 residue of the G9a ANK domain non-cage histone $\mathrm{H} 3$ binding surface can potentially contribute to $\mathrm{H} 3$ substrate recognition by the SET domain.
G9a, but not its ankyrin repeat histone binding surface, is required for the silencing of the embryonic stem cellspecific SSEA-1 surface marker during differentiation Since G9a depletion or mutation of the G9a D905 ANK non-cage residue results in deficient de novo DNA methylation of Oct3/4 during mESC differentiation, we further investigated whether this was accompanied by impaired transcriptional repression of Oct3/4 in response to RA 
treatment. To answer this question, we performed an immunoblot analysis with an antibody against Oct3/4 protein using whole cell extracts derived from mESCs that were treated with RA for the indicated number of days (Figure 2A). Interestingly, the combined defects in both global H3K9 methylation levels and de novo DNA methylation observed in the G9a-null and G9a D905R mutant mESCs do not result in impaired repression of Oct3/4 protein expression in response to RA treatment (Figure 2A). Although the G9a protein levels appear to decrease after 8 days of RA treatment for the D905R mutant but not for the TT2 control (Figure 2A), this difference was not observed in repeat experiments. We further monitored by quantitative real-time PCR the expression of the Oct3/4, Nanog, Klf4, Dppa3 and Esrrb early embryonic genes during mESC differentiation (Figure 2B). All WT, G9a knock-out and ANK mutant stable ESC lines displayed comparable mRNA levels of the above genes prior to RA treatment and the mRNA levels of these genes was similarly decreased in response to RA in all cell lines (Figure 2B). Altogether, these data show that G9a is not required for the transcriptional repression of several early embryonic genes in response to RA. The discrepancy between G9a protein levels (Figure 1B) which appear comparable amongst the parental TT2 and the constructed cell lines and G9a mRNA levels (Figure 2B), which are 50\% lower in constructed cell lines compared to the parental cell line TT2 is due to the fact that the primers used to quantify G9a mRNA detect both G9a long and short isoforms which are both expressed at a 1:1 ratio in the TT2 cell line while cell lines constructed for the purpose of this study express solely the G9a long isoform (Figure 1B upper panel: 2 bands in TT2 lane in contrast to 1 band for all other constructed cell lines).

The levels of the ESC-specific cell-surface marker SSEA-1 have been shown to inversely correlate with the degree of ESC differentiation. By flow cytometry, we observed that WT TT2 mESCs show high expression of SSEA-1 prior to RA treatment, and that these levels gradually decrease to background levels 7 days after the onset of RA treatment, as expected due to ESC differentiation (Figure 5). In contrast, G9a-null mESCs still retain partial SSEA-1 expression even 7 days after the onset of RA treatment, consistent with differentiation defects that have been attributed to the lack of G9a [15]. These results are in agreement with previous findings that G9a is essential for development and differentiation [16]. Interestingly, re-expression of WT as well as ankyrin cage and non-cage mutants of G9a rescued the impaired repression of SSEA-1 expression observed in the G9a-null ESCs (Figure 5). Indeed, all ESC lines analyzed, except the G9a-null line, show high expression of SSEA-1 prior to RA treatment and these levels gradually decrease to background levels 7 days after the onset of RA treatment. Altogether, these

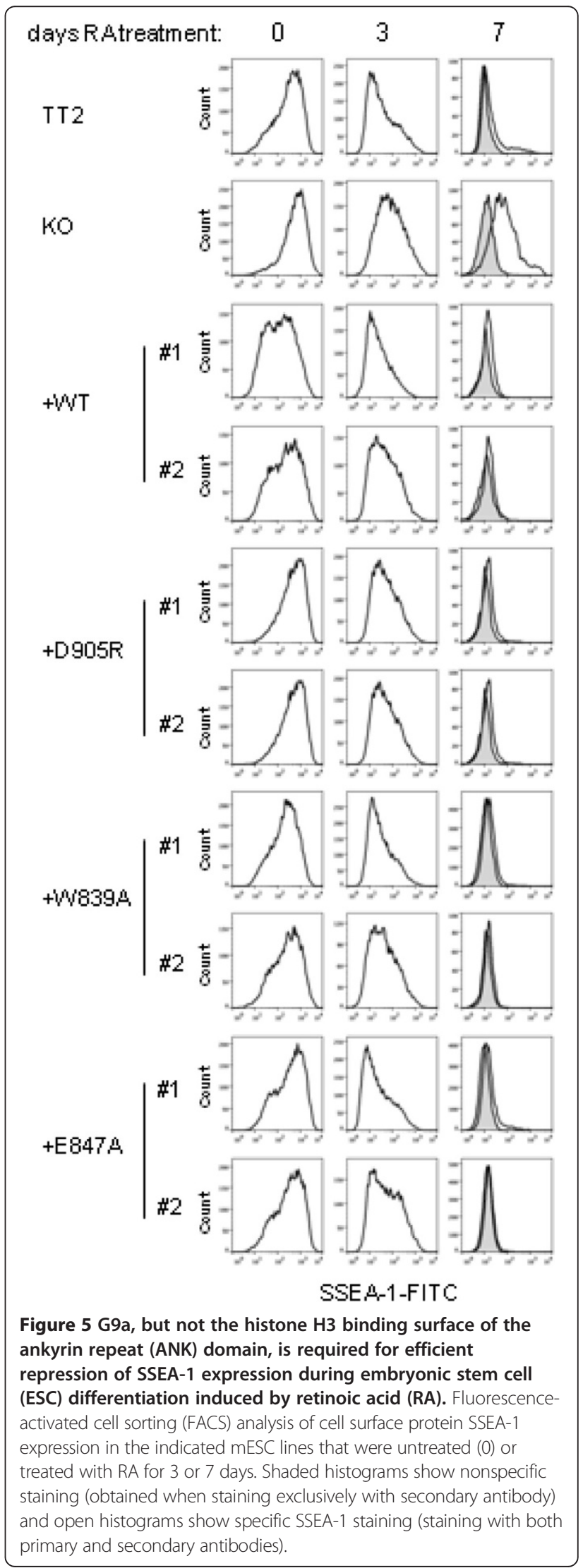


data suggest that domains or surfaces of G9a other than the histone $\mathrm{H} 3$ interaction surface of its ANK domain are required for shutting down expression of SSEA-1. In addition, the results indicate that G9a is only required for repression of a subset of the ESC genes that are turned off during the differentiation process.

\section{Discussion}

Distinct roles for the cage and non-cage regions of the G9a ankyrin repeat surface that binds K9-methylated histone $\mathrm{H} 3$

Our previous study demonstrated that the G9a ANK domain binds preferentially to histone $\mathrm{H} 3$ when it is modified by mono- or dimethylation at $\mathrm{K} 9$. In addition, we demonstrated that all of the ANK point mutations tested here disrupt binding to K9-monomethylated or -dimethylated histone H3. These mutations therefore defined a binding surface for histone H3 mono- or dimethylated at K9. The X-ray crystal structure indicated that the binding surface consists of a partial hydrophobic cage that encloses the dimethylamino moiety of the $\mathrm{K} 9$ residue of histone $\mathrm{H} 3$, and a surface outside of that cage that interacts with histone $\mathrm{H} 3$ residues 10 to 13 (Figure 1A, bottom panel). Surprisingly, the current study found that de novo DNA methylation of the Oct3/4 promoter during RA-induced mESC differentiation was prevented only by the mutation of the D905 in the non-cage portion of histone H3 binding surface, while mutation of the cage residues had no effect on the de novo DNA methylation of Oct3/4 (Figures 3B and 3D). These interesting results indicate that, while both the cage and non-cage residues are involved in binding of K9-methylated histone H3, the noncage portion of the surface has a distinct function, which presumably involves binding of a yet unknown protein that mediates the role of G9a in de novo DNA methylation of the Oct3/4 promoter. Although the percentage of DNA methylation at day $8 \mathrm{RA}$ seen in the KO (Figure 3B; 26\%) is higher than what was previously published by Epsztejn and colleagues, the percentage of DNA methylation we observed in the $\mathrm{KO}$ is consistently lower than both TT2 and +WT. We cannot rule out, however, that the phenotypes observed in the G9a D905R mutant may be due to a dominant gain of function rather than loss of function, and further studies would be required to investigate this. Importantly, our protein-protein interaction studies (Figures 4A and 4D) show that D905R mutant G9a maintains its ability to interact with both GLP and DNMT3A, therefore eliminating the possibility that the impaired DNA methylation could be due to absence of G9a-GLP complex formation or abrogated interaction with DNMT3A. Although the Asp905 residue of the G9a ankyrin domain is conserved in GLP, the phenotypes we have observed indicate that the Asp905 surface of the G9a ankyrin domain is non-redundant for its role in DNA methylation and cannot be compensated by the presence of wild-type GLP. This phenotype is in line with previous studies regarding G9a/GLP global H3K9 methylation: although both G9a and GLP conserved SET domains have histone methyltransferase activity, only the G9a SET domain (and not the GLP SET domain) catalytic function is required for H3K9 global methylation [10].

\section{Roles of the histone $\mathrm{H} 3$ binding surface and the DNMT3A binding surface of G9a in de novo DNA methylation of the Oct3/4 promoter}

The ability of G9a ANK domain to bind DNMT3A provides a potential mechanism for the G9a-dependent de novo DNA methylation of pluripotency genes such as Oct3/4 during mESC differentiation; that is, G9a could recruit DNMT3A to the Oct3/4 promoter. One way to test this hypothesis is to identify the DNMT3A binding surface of the G9a ANK domain and make point mutations that eliminate DNMT3A binding. However, we found that the G9a protein interaction surface that binds histone $\mathrm{H} 3$ methylated at $\mathrm{K} 9$ is not involved in the binding of DNMT3A by G9a. Thus, DNMT3A binds to a yet undefined surface of the G9a ANK domain. Furthermore, since the D905R mutation in the histone H3 binding surface eliminated G9a-dependent de novo DNA methylation of the Oct 4 promoter without affecting the G9a-DNMT3A interaction, our results indicate that the ability of G9a to bind DNMT3A is not, by itself, sufficient to explain G9a-dependent de novo DNA methylation. Rather, while it still seems reasonable to assume that the binding of DNMT3A is relevant for G9a-dependent de novo DNA methylation, the ability of G9a to bind histone H3 or a yet unknown protein through the binding surface that includes D905 is also required.

\section{A novel role for the ankyrin repeat domain in G9a-dependent H3K9 methylation}

Our studies have shown that G9a binding to H3K9me2 through the partial hydrophobic cage surface of the ANK domain is not required for the maintenance of global H3K9me2 levels. These results are surprising in the light of the fact that it has been thought that G9a binding to methyl-H3K9 might assist in propagating/ spreading this methyl mark across genomic regions and thus might contribute to global cellular H3K9me2 levels. Data presented here do not support this hypothesis and leave open the question of the physiological relevance of G9a ANK binding to methyl-H3K9. In contrast, the D905R mutation not only prevented the G9a-dependent de novo DNA methylation during RA-induced differentiation of mESC, but also caused a dramatic reduction in global cellular levels of $\mathrm{H} 3 \mathrm{~K} 9 \mathrm{me} 2$ in the undifferentiated mESC. Both of these phenotypes mirror that of mESC lacking G9a entirely, indicating that this ANK surface is 
critically important for both of these G9a functions. Importantly, the impaired DNA methylation observed in the G9a D905R ESCs is likely not due to defects in the formation of the G9a-GLP heteromeric complex since the D905R G9a mutation does not abrogate the interaction between G9a and GLP. Notably, although G9a-like protein (GLP) also has a SET domain harboring H3K9 methyltransferase activity, it is not surprising that the deficient H3K9me2 levels resulting from the G9a D905R mutation cannot be compensated by GLP since it has previously been shown that the catalytic activity of GLP is dispensable for H3K9 methylation in vivo [10].

As with de novo DNA methylation of the Oct3/4 promoter, only the non-cage mutation D905R, but not any of the cage mutations, reduced cellular H3K9me2 levels. Thus, surprisingly these results indicate a critical role for the ANK domain in establishing and/or maintaining the global H3K9me2 levels. While the protein interaction by the non-cage ANK surface that is required to support H3K9 methylation by G9a has not been determined, the fact that this non-cage surface interacts with residues 10 to 13 of histone $\mathrm{H} 3$ in the previously published X-ray crystal structure suggests that this domain may somehow cooperate with the SET domain in recognizing and/or binding the $\mathrm{H} 3$ substrate for methylation by the SET domain. Although we did not detect ANK binding to unmethylated histone $\mathrm{H} 3$ in vitro [11], this does not rule out the possibility that the non-cage ANK surface could participate in an interaction with unmethylated histone H3 in conjunction with some other protein or G9a domain that binds unmethylated histone $\mathrm{H} 3$, such as the G9a SET domain. Moreover, while the SET domain alone is capable of methylating histone $\mathrm{H} 3$ in vitro, additional experiments are required to test whether the ANK domain and the non-cage histone $\mathrm{H} 3$ binding surface somehow enhance the enzymatic function of G9a.

\section{Roles of G9a in retinoic acid-induced differentiation of mouse embryonic stem cells}

Previous studies demonstrated that G9a is required for mESC differentiation [15]. However, while de novo DNA methylation of the Oct3/4 promoter fails to occur in $69 a$ null mESC, Oct3/4 and Nanog gene expression is still repressed in these cells after RA treatment, and other specific molecular drivers and markers of the differentiation process that require G9a were not identified. By examining an expanded list of $\mathrm{mESC}$-specific genes, we found that, in addition to Oct3/4 and Nanog, G9a is also not required for repression of $K l f 4, E s r r b$ and Dppa3 during RA-induced differentiation. Therefore, other histone modifiers (such as the PRC complex, for instance) and chromatin-remodeling proteins are most likely involved in the repression of these genes during ESC differentiation. In contrast, we found that repression of SSEA-1 is compromised by absence of G9a. Thus, G9a is required selectively for repression of $\mathrm{mESC}$-specific genes, and SSEA-1 represents the first identified mESC-specific protein marker that requires G9a for its repression during RA-induced differentiation. This represents a valuable marker of G9a-mediated ESC differentiation defects that is both quantifiable and not lineage-specific. In addition, we found that none of the point mutations in the ANK surface that bind histone H3 prevented G9a-dependent repression of SSEA-1 expression, indicating that G9a domains outside of this histone $\mathrm{H} 3$-binding region of the ANK domain are responsible for repression of SSEA-1 by G9a during RA-induced differentiation. Furthermore, the fact that cell lines expressing D905R mutant G9a have compromised histone H3K9 and DNA methylation and yet retain the ability to repress SSEA-1 expression in response to RA suggests that histone H3K9 and DNA methylation are not required for early steps of ESC differentiation. However, these activities may be required to achieve full terminal differentiation.

\section{Conclusions}

Our data validate previous findings suggesting that the G9a ANK domain is required for its role in de novo DNA methylation of $\operatorname{Oct} 3 / 4$, and we define a specific residue (Asp905) of the ANK domain as a key interaction surface likely involved in protein-protein interactions critical for G9a role in DNA methylation. Importantly, these findings open exciting avenues for investigating the protein(s) that interact(s) with G9a Asp905 in order to gain a better understanding of the precise molecular mechanisms of G9a in de novo DNA methylation. In addition, our data show that the G9a ANK domain methylhistone binding function is not required for its role in DNA methylation of Oct3/4 during ESC differentiation. Altogether, the results presented in this report advance our current understanding of the G9a ANK domain structure and distinct functional surfaces, revealing an unexpected role of the ANK domain both in global histone H3K9 methylation in undifferentiated ESCs and in de novo DNA methylation in a manner independent of the interaction between G9a and DNMT3A. Furthermore, these data collectively contribute to us gaining better insights into the molecular mechanisms (domains, surfaces and protein interactions) of G9a involved in ESC self-renewal and differentiation. Since epigenetic modifiers are increasingly being valued as promising targets for cancer drugs, it is important to investigate the role of G9a in ESC differentiation as a clearer understanding of this process would enable development of safer and more efficient reprogramming strategies for future therapeutic applications of stem cells, and shed light on fundamental questions concerning the establishment of cellular identity. 


\section{Methods}

\section{Mouse embryonic stem cell culture}

TT2 line and G9a-null ES cells were provided by Dr. Yoichi Shinkai (Kyoto University, Kyoto, Japan). Undifferentiated ES cells were maintained in Dulbecco's Modified Eagle's medium (DMEM, GIBCO) supplemented with 13\% (vol/vol) Stem Cell Qualified FBS (Gemini), $100 \mu \mathrm{M}$ nonessential amino acids (GIBCO), $100 \mu \mathrm{M}$ 2-mercaptoethanol (Sigma) and $10^{3} \mathrm{U} / \mathrm{ml}$ leukemia inhibitory factor (LIF; Millipore) at $37^{\circ} \mathrm{C}$, and $5 \% \mathrm{CO} 2$. ESCs were seeded on gelatin (Millipore)-coated dishes. For retinoic-acid (RA)-induced differentiation, cells were cultured in the presence of $1 \mu \mathrm{M}$ all-trans-RA (Sigma) without LIF.

\section{Generation of G9a-mutant embryonic stem cells}

To generate stable cell lines expressing wild-type (WT) or mutant G9a, the pHRCMVpuro-Sin8 vector containing a puromycin resistance cassette was introduced simultaneously with a threefold excess molar ratio of the G9a expression vector into the G9a-null ES cells by either standard electroporation or by nucleofection. Nucleofection ( $1 \mu \mathrm{g}$ of plasmid DNA per $10^{6}$ cells) was performed using the Amaxa 4-D Nucleofector System (Lonza) with the P3 Primary Cell 4-D Nucleofector X Solution and the CG-104 program. Plasmid DNA used for nucleofection was purified using the Endotoxin-free Plasmid Maxiprep Kit (Qiagen) according to the manufacturer's instructions. The stable transfectant clones expressing mutant or WT G9a were selected in ESC medium containing puromycin $(1 \mu \mathrm{g} / \mathrm{ml})$. All mutations were confirmed in the selected cell clones by sequencing.

\section{Plasmids}

The following mammalian expression vectors were described in previous publications: pSG5-FLAG encoding full-length mouse G9a protein, including wild-type, ankyrin mutants (D905R, E870R, W839A, E847A and W877A) and SET mutant (H1166K) [11]; pcDNA3.1-mDNMT3A [17]; and pHRCMVpuro-Sin8 [18]; pSG5-HA encoding full length mouse $\mathrm{G} 9 \mathrm{a}$ and a deletion mutant $(\triangle \mathrm{ANK})$ lacking the ankyrin repeat domain (amino acids 734 to 934) [19]. The GLP expression vector pSG5-FLAG GLP (full-length, wild-type) was generated by PCR.

\section{Antibodies used for immunoblot}

The following antibodies were purchased and used for this study: antibody to G9a (PP-A8620A-00) from R\&D Biosystems; antibody to DNMT1 (IMG-261A) from Imgenex; antibody to HA (11867431001) from Roche; antibodies to H3K9me2 (ab1220), DNMT3A (ab2850), and DNMT3B (ab2851) from Abcam; antibodies to Oct3/4 (sc-8629), and H3 (sc-10809) from Santa Cruz Biotechnology; antibodies to FLAG (F1804), and GAPDH (G9545) from Sigma; antibodies to Sox2 (AB5603), and Nanog (AB5731) from Millipore. Immunoblot detection was performed by chemiluminescence using Super Signal West Dura (Thermo Scientific) for proteins with low expression levels and HyGLO (Denville Scientific) for all other proteins according to the manufacturer's instructions. Immunoblots were performed on whole cell extracts (RIPA buffer) or Acid Histone Extracts (http://www.abcam.com/ protocols/histone-extraction-protocol-for-western-blot).

\section{Real-time RT-qPCR analysis}

Total RNA was isolated using TRIzol (Invitrogen) according to the manufacturer's instructions. Reverse transcription was performed by using iScript Advanced cDNA Synthesis Kit for RT-qPCR (BioRad) according to the manufacturer's instructions by using $5 \mu \mathrm{g}$ of total RNA as template. qPCR amplification was performed on a Roche LightCycler 480 by using SYBR Green I Master Mix (Roche) and the following PCR primers: G9a.Fwd (5' AGCTTCGGAACAAAGAAGGAG -3') and G9a.Rev (5' - ACAGGGGATGGGTACATTCTC -3'); UBC.Fwd (5' - CAGCCGTATATCTTCCCAGACT - $3^{\prime}$ ) and UBC. Rev (5' - CTCAGAGGGATGCCAGTAATCTA -3'); Oct3/ 4.Fwd (5' - CTGAAGCAGAAGAGGATCACC -3') and Oct3/4.Rev (5' - GCCGCAGCTTACACATGTTC -3'); Nanog.Fwd (5' - CAGCCTCCAGCAGATGCAAG -3') and Nanog.Rev (5' - AATCAGACCATTGCTAGTCTT C - 3'); Klf4.Fwd (5' - TCTCAAGGCACACCTGCGA AC - $3^{\prime}$ ) and Klf4.Rev (5'- GGTAGTGCCTGGTCAG TTCATC -3'); Esrrb.Fwd (5' - CGCAAGAGCTACGA GGACTG -3') and Esrrb.Rev (5'- GGTAGCCAGAG GCAATGTCC -3'); and Dppa3.Fwd (5' - AGTCTACG GAACCGCATTGC -3') and Dppa3.Rev (5'- GCTA TAGGGTCTTTCAGCACCG -3').

\section{Bisulfite gDNA sequencing}

Genomic DNA was isolated from ESC lines that were untreated or treated with RA for 8 days using the DNeasy Blood and Tissue Kit (Qiagen) according to the manufacturer's instructions. Bisulfite treatment of the genomic DNA was performed using the EZ DNA Methylation Kit (Zymo Research) according to the manufacturer's instructions. PCR was performed to amplify the Oct3/4 promoter with the following primers: Oct3/4.bs.F (5'- TGGGTT GAAATATTGGGTTTATTT -3') and Oct3/4.bs.R (5'- C TAAAACCAAATATCCAACCATA -3'). PCR products were subcloned into $\mathrm{pCR}$ 2.1-TOPO vector using the TOPO TA Cloning Kit (Invitrogen) and sequencing was performed using the M13R primer that localizes to the vector backbone.

\section{Transfection co-immunoprecipitation}

COS - 7 cells were transiently transfected by using Lipofectamine 2000 (Invitrogen) with $5 \mu \mathrm{g}$ of plasmid encoding 
G9a and $1 \mu \mathrm{g}$ of plasmid encoding DNMT3A or, alternatively, $4 \mu \mathrm{g}$ of plasmid encoding GLP. At $72 \mathrm{~h}$ after transfection, cell extracts were prepared in RIPA buffer with Nonidet P-40 and sodium deoxycholate replaced by Triton X-100 (50 mM Tris- $\mathrm{HCl}$ (pH 8.0), $150 \mathrm{mM} \mathrm{NaCl}$, $1 \%$ Triton X-100, and $1 \mathrm{mM}$ EDTA). Antibodies used were the same as those indicated for immunoblots.

\section{Fluorescence-activated cell sorting analysis and cell sorting} Cells were trypsinized, washed once in PBS, and resuspended in PBS; dead cells were stained using the LIVE/ DEAD Fixable Dead Cell Stain Kit (Invitrogen) according to the manufacturer's instructions. Next, cells were fixed in 2\% paraformaldehyde (PFA) for $15 \mathrm{~min}$ at room temperature, washed with PBS and stained with antiSSEA-1 mouse monoclonal IgM antibody (mc-480, University of Iowa, Developmental Studies Hybridoma Bank, $1: 400)$ at $37^{\circ} \mathrm{C}$ for $20 \mathrm{~min}$. After a PBS wash, the cells were labeled with fluorescein isothiocyanate (FITC)conjugated goat anti-mouse IgM (Millipore, 1:200) for 15 min at $37^{\circ} \mathrm{C}$. After one PBS wash, stained cells were resuspended in PBS containing 1\% FBS and 3\% BSA, and at least 10,000 events of healthy cells were analyzed with a CyAN Analyzer (Beckman Coulter) and Flowjo ver.10 software (Tree Star, Ashland, USA). Control cells were not treated with primary antibody.

\footnotetext{
Abbreviations

ANK: ankyrin repeat; ESC: embryonic stem cell; KO: knock-out; HA: human influenza hemagglutinin; WT: wild-type; mRNA: messenger RNA; FACS: fluorescence-activated cell sorting; H3K9: histone H3 lysine K9; RA: retinoic acid; SSEA-1: stage-specific embryonic antigen 1.
}

\section{Competing interests}

The authors declare that they have no competing interests.

\section{Authors' contributions}

DB supervised the project, conceived strategies, designed and performed experiments, analyzed data and wrote the paper; BHL. performed experiments and analyzed data; LG performed experiments and analyzed data; DSG. performed experiments and analyzed data; M.R.S. supervised the project and wrote the paper. All authors read and approved the final manuscript.

\section{Acknowledgements}

We thank the following colleagues at the University of Southern California: Dr. Peter A. Jones and Dr. Wange Lu for discussion and support; Dr. Colin Exline, Dr. Peilin Zhang, Dr. Sewoon Kim and Dr. Jueng Soo You for experimental protocols and technical advice; and Laurie Herviou for technical assistance. We thank Dr. Yoichi Shinkai (Kyoto University) for the gift of the T2 and G9a-null mouse embryonic stem cells; Dr. Jeffrey P. Northrop (University of British Columbia) for G9a expression plasmids and heterogenous mouse embryonic cell populations expressing wild-type G9a or G9a harboring mutations in the ankyrin and SET domains; Dr. Shoji Tajima (University of Osaka) for the DNMT3A expression plasmid used for transfection co-immunoprecipitation; and Dr. Corentin Laulier (City of Hope) and Dr. Daniel de Carvalho (University of Toronto) for experimental protocols and technical advice. FACS data was generated at the Flow Cytometry Core of the USC Norris Comprehensive Cancer Center and analyzed with support from Lora Barsky. This work was supported by the National Institutes of Health (DK055274 to M.R.S.); the National Cancer Institute (Cancer Center Support Grant P30CA014089 to the University of Southern California); and the California Institute of Regenerative Medicine postdoctoral training grant to D.B.
Received: 9 January 2014 Accepted: 9 September 2014

Published: 22 October 2014

\section{References}

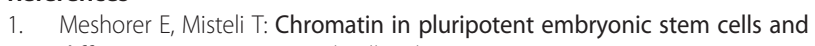
differentiation. Nat Rev Mol Cell Biol 2006, 7:540-546.

2. Hawkins RD, Hon GC, Lee LK, Ngo Q, Lister R, Pelizzola M, Edsall LE, Kuan S, Luu Y, Klugman S, Antosiewicz-Bourget J, Ye Z, Espinoza C, Agarwahl S, Shen L, Ruotti V, Wang W, Stewart R, Thomson JA, Ecker JR, Ren B: Distinct epigenomic landscapes of pluripotent and lineage-committed human cells. Cell Stem Cell 2010, 6:479-491.

3. Wen $B$, Wu H, Shinkai Y, Irizarry RA, Feinberg AP: Large histone H3 lysine 9 dimethylated chromatin blocks distinguish differentiated from embryonic stem cells. Nat Genet 2009, 41:246-250.

4. Efroni S, Duttagupta R, Cheng J, Dehghani H, Hoeppner DJ, Dash C, BazettJones DP, Le Grice S, McKay RD, Buetow KH, Gingeras TR, Misteli T, Meshorer E: Global transcription in pluripotent embryonic stem cells. Cell Stem Cell 2008, 2:437-447.

5. Xie W, Schultz MD, Lister R, Hou Z, Rajagopal N, Ray P, Whitaker JW, Tian S, Hawkins RD, Leung D, Yang H, Wang T, Lee AY, Swanson SA, Zhang J, Zhu Y, Kim A, Nery JR, Urich MA, Kuan S, Yen CA, Klugman S, Yu P, Suknuntha K, Propson NE, Chen H, Edsall LE, Wagner U, Li Y, Ye Z, et al: Epigenomic analysis of multilineage differentiation of human embryonic stem cells. Cell 2013, 153:1134-1148.

6. Tachibana M, Sugimoto K, Nozaki M, Ueda J, Ohta T, Ohki M, Fukuda M, Takeda N, Niida H, Kato H, Shinkai Y: G9a histone methyltransferase plays a dominant role in euchromatic histone $\mathrm{H} 3$ lysine 9 methylation and is essential for early embryogenesis. Genes Dev 2002, 16:1779-1791.

7. Epsztejn-Litman S, Feldman N, Abu-Remaileh M, Shufaro Y, Gerson A, Ueda J, Deplus R, Fuks F, Shinkai Y, Cedar H, Bergman Y: De novo DNA methylation promoted by G9a prevents reprogramming of embryonically silenced genes. Nat Struct Mol Biol 2008, 15:1176-1183

8. Feldman N, Gerson A, Fang J, Li E, Zhang Y, Shinkai Y, Cedar H, Bergman Y: G9a-mediated irreversible epigenetic inactivation of Oct-3/4 during early embryogenesis. Nat Cell Biol 2006, 8:188-194.

9. Athanasiadou R, de Sousa D, Myant K, Merusi C, Stancheva I, Bird A: Targeting of de novo DNA methylation throughout the Oct-4 gene regulatory region in differentiating embryonic stem cells. PLoS One 2010, 5:e9937.

10. Tachibana M, Matsumura Y, Fukuda M, Kimura H, Shinkai Y: G9a/GLP complexes independently mediate $\mathrm{H} 3 \mathrm{~K} 9$ and DNA methylation to silence transcription. Embo J 2008, 27:2681-2690.

11. Collins RE, Northrop JP, Horton JR, Lee DY, Zhang X, Stallcup MR, Cheng X: The ankyrin repeats of G9a and GLP histone methyltransferases are mono- and dimethyllysine binding modules. Nat Struct Mol Biol 2008, 15:245-250

12. Dong KB, Maksakova IA, Mohn F, Leung D, Appanah R, Lee S, Yang HW, Lam LL, Mager DL, Schubeler D, Tachibana M, Shinkai Y, Lorincz MC: DNA methylation in ES cells requires the lysine methyltransferase G9a but not its catalytic activity. EMBO J 2008, 27:2691-2701.

13. Tamaru H, Selker EU: A histone $\mathrm{H} 3$ methyltransferase controls DNA methylation in Neurospora crassa. Nature 2001, 414:277-283.

14. Tachibana M, Ueda J, Fukuda M, Takeda N, Ohta T, Iwanari H, Sakihama T, Kodama T, Hamakubo T, Shinkai Y: Histone methyltransferases G9a and GLP form heteromeric complexes and are both crucial for methylation of euchromatin at H3-K9. Genes Dev 2005, 19:815-826.

15. Melcer S, Hezroni H, Rand E, Nissim-Rafinia M, Skoultchi A, Stewart CL, Bustin M, Meshorer E: Histone modifications and lamin A regulate chromatin protein dynamics in early embryonic stem cell differentiation. Nat Commun 2012, 3:910.

16. Tachibana M, Sugimoto K, Fukushima T, Shinkai Y: Set domain-containing protein, G9a, is a novel lysine-preferring mammalian histone methyltransferase with hyperactivity and specific selectivity to lysines 9 and 27 of histone H3. J Biol Chem 2001, 276:25309-25317.

17. Suetake I, Shinozaki F, Miyagawa J, Takeshima H, Tajima S: DNMT3L stimulates the DNA methylation activity of Dnmt3a and Dnmt3b through a direct interaction. J Biol Chem 2004, 279:27816-27823. 
18. Ou CY, Kim JH, Yang CK, Stallcup MR: Requirement of cell cycle and apoptosis regulator 1 for target gene activation by Wnt and beta-catenin and for anchorage-independent growth of human colon carcinoma cells. J Biol Chem 2009, 284:20629-20637.

19. Purcell DJ, Jeong KW, Bittencourt D, Gerke DS, Stallcup MR: A distinct mechanism for coactivator versus corepressor function by histone methyltransferase G9a in transcriptional regulation. J Biol Chem 2011, 286:41963-41971.

doi:10.1186/1756-8935-7-27

Cite this article as: Bittencourt et al:: Role of distinct surfaces of the G9a ankyrin repeat domain in histone and DNA methylation during embryonic stem cell self-renewal and differentiation. Epigenetics \& Chromatin 2014 7:27.

\section{Submit your next manuscript to BioMed Central and take full advantage of:}

- Convenient online submission

- Thorough peer review

- No space constraints or color figure charges

- Immediate publication on acceptance

- Inclusion in PubMed, CAS, Scopus and Google Scholar

- Research which is freely available for redistribution 